\title{
Technical considerations in transforaminal endoscopic spine surgery at the thoracolumbar junction: report of 3 cases
}

\author{
Albert E. Telfeian, MD, PhD, ${ }^{1}$ Gabriele P. Jasper, MD, ${ }^{2}$ Adetokunbo A. Oyelese, MD, $\mathrm{PhD},{ }^{1}$ and \\ Ziya L. Gokaslan, MD'
}

${ }^{1}$ Department of Neurosurgery, Rhode Island Hospital, The Warren Alpert Medical School of Brown University, Providence, Rhode Island; and ${ }^{2}$ Center for Pain Control, Brick, New Jersey

\begin{abstract}
OBJECTIVE In this study the authors describe the technical considerations and feasibility of transforaminal discectomy and foraminoplasty for the treatment of lumbar radiculopathy in patients who have herniated discs at the thoracolumbar junction.
\end{abstract}

METHODS After institutional review board approval, charts from 3 consecutive patients with lumbar radiculopathy and T12-L1 herniated discs who underwent endoscopic procedures between 2006 and 2014 were reviewed.

RESULTS Consecutive cases $(n=1316)$ were reviewed to determine the incidence and success of surgery performed at the T12-L1 level. Only 3 patients $(0.23 \%)$ treated with endoscopic surgery for their herniated discs had T12-L1 herniated discs; the rest were lumbar or lumbosacral herniations. For patients with T12-L1 disc herniations, the average preoperative visual analog scale score was 8.3 (indicated in the questionnaire as describing severe and constant pain). The average 1-year postoperative visual analog scale score was 1.7 (indicated in the questionnaire as mild and intermittent pain).

CONCLUSIONS Transforaminal endoscopic discectomy and foraminotomy can be used as a safe yet minimally invasive technique for the treatment of lumbar radiculopathy in the setting of a thoracolumbar disc herniation.

http://thejns.org/doi/abs/10.3171/2015.10.FOCUS15372

KEY WORDS endoscopic discectomy; minimally invasive; transforaminal; thoracolumbar

$\mathrm{I}$ NTERVERTEBRAL disc herniations at the thoracolumbar junction (T12-L1, L1-2, and L2-3) make up only approximately $1 \%-2 \%$ of lumbar disc herniations, ${ }^{4,10}$ which may be due to the decreased motion occurring at this area of the spinal column. The reported outcomes for discectomy surgery at the thoracolumbar junction are inferior to those reported in the lower lumbar spine. The worse outcomes for thoracolumbar disc surgery may be related to the anatomical features peculiar to the thoracolumbar region. The narrow space between the two partes interarticulares and the proximity of the conus medullaris makes surgery at the thoracolumbar junction for a herniated disc a fundamentally different operation.

Transforaminal endoscopic lumbar discectomy is a minimally invasive spinal surgery procedure that was introduced by Kambin and Gellman in 1973, as detailed in their subsequent paper. ${ }^{5}$ Advances in endoscopic visualization and instrumentation as well as increased patient demand for more minimally invasive procedures have led to an increased popularity of the technique, particularly outside the US. In this report we describe our experience with endoscopic discectomy and foraminotomy in the treatment of patients with thoracolumbar herniated discs. These are patients who present with persistent lumbar radiculopathy despite conservative nonoperative treatment. We report a retrospective study on the average pain relief 1 year after endoscopic discectomy and foraminotomy in patients with T12-L1 disc herniations. Transforaminal endoscopic discectomy and foraminotomy is described here as an ultra-minimally invasive solution to the problem of lumbar radiculopathy in the setting of a thoracolumbar herniated disc. 


\section{Methods \\ Review of Cases}

After institutional review board approval, charts from 3 consecutive patients (mean age 58.3 years, 1 woman and 2 men) with lumbar radiculopathy and T12-L1 disc herniations who underwent endoscopic procedures between 2006 and 2014 were reviewed. Data are only presented for patients who underwent single-level, unilateral endoscopic decompression at the T12-L1 level-no cases of multilevel, bilateral, or decompressions adjacent to a fusion are presented.

All 3 patients with T12-L1 disc herniations presented with severe thigh pain and sensory disturbance at the anterior aspect of the thigh. Two patients complained of groin pain. There were no clear signs of lower-extremity weakness, muscle atrophy, or loss of deep tendon reflex, and there were no complaints of bowel or bladder dysfunction in these patients.

\section{Operative Technique}

Patients were selected for treatment based on the results of their MRI, physical examination, and dermatomal pain pattern. All patients who were considered for endoscopic surgical treatment had already exhausted more conservative treatments, which included but were not limited to physical therapy and epidural steroid injections.

Patients were positioned prone on the Wilson frame or in the lateral decubitus position with the operating room table reversed and the flank over the break in the table. The choice of patient position was based on both the surgeon's and the patient's preference. The procedure was done under local analgesia and intravenous sedation (other practitioners do perform this procedure with general anesthesia); the level of anesthetic was titrated so that the patient was able to communicate with the surgeon throughout the procedure. The joimax TESSYS endoscopic system was used for the procedure. Percutaneous entry was established, entering through the skin 5-8 cm lateral to the midline. Using intermittent fluoroscopic guidance, alternating between a lateral and anteroposterior (AP) view, a $25-\mathrm{cm} 18$-gauge needle was advanced and placed in the disc space through Kambin's triangle, between the exiting and traversing nerves. An AP fluoroscopic view was used to assure that the disc space was entered before the needle was past the middle of pedicle. Sequential reamers were used to enlarge the neural foramen by removing the ventral aspect of the superior facet.

Discectomy was performed with straight, up-going, and bendable graspers (Fig. 1D, 1G, and Video 1).

VIDEO 1. Clip showing a T12-L1 endoscopic discectomy. Copyright Albert Telfeian. Published with permission. Click here to view.

After foraminotomy, the semibendable grasper could be observed reaching under the thecal sac in the epidural space with endoscopic and fluoroscopic visualization (Fig. $1 G)$. By rotating the beveled cannula working channel and endoscope, a $360^{\circ}$ visualization of the anulus and exiting and traversing nerve roots was possible. The technical success of the foraminotomy procedure was determined by the visualization of the exiting and traversing nerve root and visualizing the ball-tip probe dilator (Fig. 1C) passing freely under the nerve and over the inferior pedicle. After adequate discectomy and foraminotomy, prior to terminating the procedure the patient was asked about the status of his or her radicular symptoms. The working channel and scope were removed, pressure was held on the 5 -mm incision for 5 minutes, and the wound was closed with a single interrupted suture. Patients were discharged between 30 and 60 minutes after the procedure.

\section{Results}

In a series of 1316 patients, 3 (only $0.23 \%$ ) underwent single-level endoscopic discectomy and foraminotomy at T12-L1. The average pain relief 1 year postoperatively after endoscopic treatment was reported to be $80 \%$. The average preoperative visual analog scale (VAS) score was 8.3 , indicated in our questionnaire as severe and constant pain. The average 1-year postoperative VAS score was 1.7, indicated in our questionnaire as mild and intermittent pain.

There were no reports of infection, dural tear, thrombophlebitis, spinal instability, or renal, vascular, or pulmonary injury. There were no serious complications such as cauda equina syndrome or nerve damage resulting in paralysis. There were no issues with postoperative instability during the 1-year follow-up, but surveillance flexion-extension radiographs were not performed. Previously reported complications can include infection, dysesthesia, thrombophlebitis, dural tear, vascular injury, and death. ${ }^{8}$

\section{Discussion}

Before treating T12-L1 disc herniations via a transforaminal endoscopic approach, 3 technical challenges were considered: 1) proximity of the kidney; 2) proximity of the rib; and 3) thecal sac/spinal canal diameter ratio. Preoperative planning was similar to that used in other stereotactic neurosurgery procedures. A safe corridor was mapped on the preoperative axial MR images to avoid the kidney and rib. Special consideration was given to the thecal sac/ spinal canal diameter ratio: several studies have demonstrated the differences in the interpedicular distance (transverse diameter of the spinal canal) at L-1 (approximately $22 \mathrm{~mm}$ ) versus L-5 (approximately $30 \mathrm{~mm}$ )., ${ }^{2,6,11}$ For transforaminal targeting at L-5, the usual target is the medial pedicle wall on AP fluoroscopy, just entering the disc on lateral fluoroscopy. This targeting would have the endoscope enter the thecal sac at T12-L1. Figure 1B shows the targeting for transforaminal endoscopic access at T12-L1: the needle trajectory is planned to enter the disc space at the midpedicle line, to get as close as possible to the herniation impinging the neural structures without directly contacting them.

The incidence of disc herniations treated at T12-L1 presented in this series is low, but may be somewhat higher than that found in the general population. Other studies of the incidence of thoracolumbar disc herniations note an incidence of $1 \%-2 \%$ for disc herniations that include T12-L1, L1-2, and L2-3., Many of the individuals in the patient population described here are referred specifically for endoscopic spine surgery because they have received other surgical opinions and want to avoid instrumented fu- 

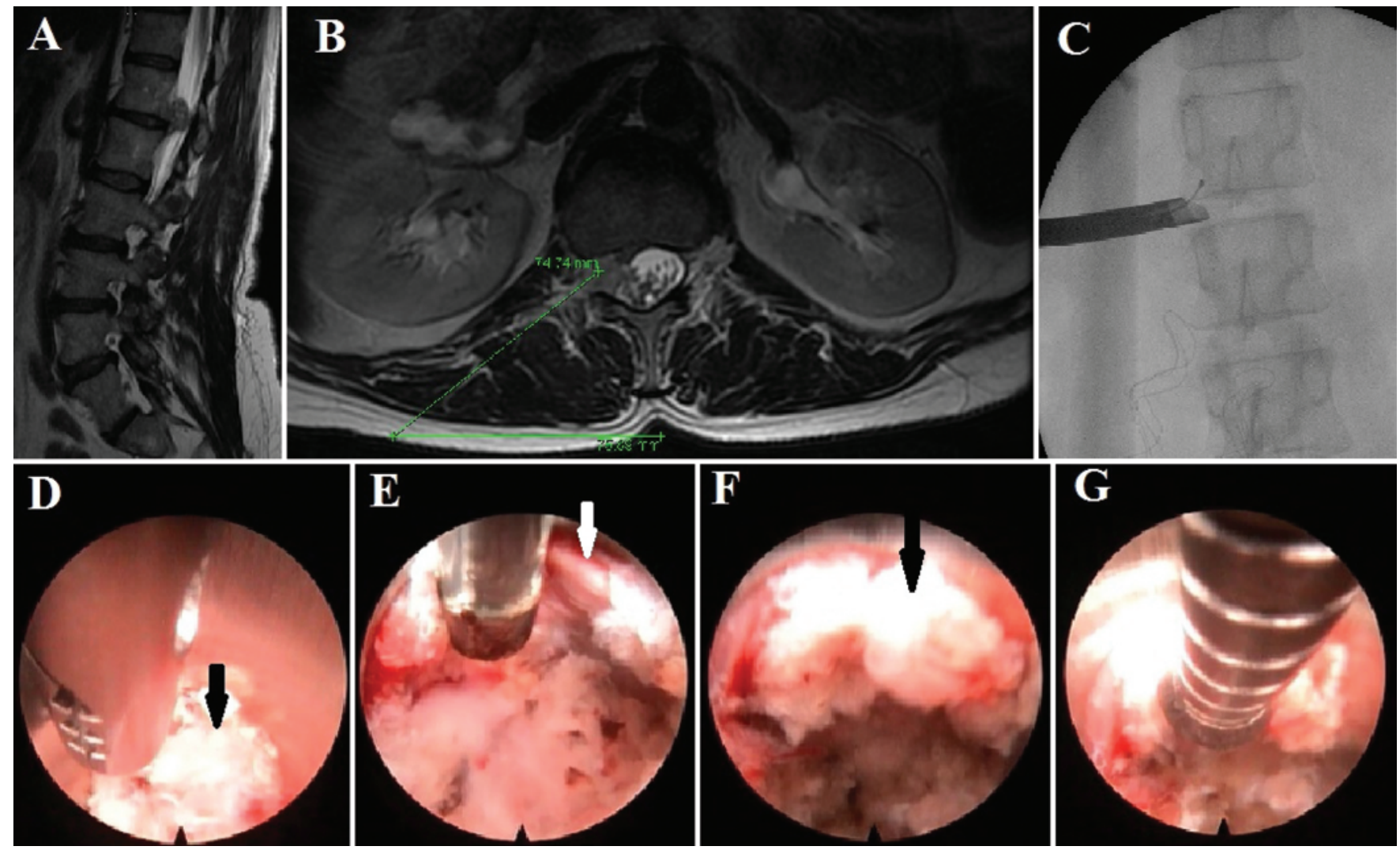

FIG. 1. A: Sagittal T2-weighted MRI study illustrating a large T12-L1 herniated disc referred for endoscopic treatment. B: Axial T2-weighted MRI study demonstrating preoperative planning to determine the safe corridor for transforaminal access to the disc herniation, avoiding the kidney, ribs, and thecal sac. C: An AP fluoroscopic view illustrating the working channel and ball-tip probe instrument in the T12-L1 neural foramen. D: Endoscopic view of the straight grasper entering into the endoscopic view at the beginning of the discectomy and the working channel docked on the herniated disc (black arrow). E: Endoscopic bipolar electrocautery demonstrating maintaining hemostasis adjacent to the thecal sac (white arrow). F: Endoscopic view of a large disc fragment extruding from under the epidural space after manipulation with the ball-tip probe (panel C). G: Endoscopic view of semibendable grasper with its articulating edges reaching under the thecal sac to remove the rest of the disc extrusion.

sion surgery. Other spine surgeons consider instrumented lumbar spine fusion procedures as the principal treatment for disc herniations at the thoracolumbar junction because a conventional posterior approach would require extensive resection of the facet joint to obtain adequate exposure of the disc fragment. ${ }^{12}$ Also to be noted, the patients presented here were selected for endoscopic spine surgery because their lesions (foraminal and paracentral disc herniations) were very accessible to the transforaminal endoscopic approach-less accessible ones (midline disc herniations or facet cysts) were excluded in this series.

Other studies have shown that endoscopic spine surgery is an effective procedure for treating multiple pathologies in the lumbar spine including lateral, paracentral, central, extruded, and even contralateral herniated discs as well as lateral recess stenosis. ${ }^{3,7,9,13}$ The success of transforaminal endoscopic surgery at T12-L1 may not be surprising given the nearly $80 \%$ excellent or good results reported in a series of 45 patients who underwent endoscopic procedures at L1-2 and L2-3 by authors in South Korea.

\section{Conclusions}

In this series, transforaminal endoscopic surgery is proposed as a novel technique in the setting of a T12L1 disc herniation. Here a transforaminal approach that avoids a posterior approach, reduces the need to remove stabilizing facet bone, does not require retraction on the conus medullaris, is truly minimally invasive, does not involve an instrumented fusion, and does not require general anesthesia is suggested as a helpful addition in the spine surgeon's minimally invasive armamentarium.

\section{References}

1. Ahn Y, Lee SH, Lee JH, Kim JU, Liu WC: Transforaminal percutaneous endoscopic lumbar discectomy for upper lumbar disc herniation: clinical outcome, prognostic factors, and technical consideration. Acta Neurochir (Wien) 151:199_ 206, 2009

2. Amonoo-Kuofi HS: Maximum and minimum lumbar interpedicular distances in normal adult Nigerians. J Anat 135:225-233, 1982

3. Choi G, Lee SH, Lokhande P, Kong BJ, Shim CS, Jung B, et al: Percutaneous endoscopic approach for highly migrated intracanal disc herniations by foraminoplastic technique using rigid working channel endoscope. Spine (Phila Pa 1976) 33:E508-E515, 2008

4. Hsu K, Zucherman J, Shea W, Kaiser J, White A, Schoffer- 
man J, et al: High lumbar disc degeneration. Incidence and etiology. Spine (Phila Pa 1976) 15:679-682, 1990

5. Kambin P, Gellman PH: Percutaneous lateral discectomy of the lumbar spine: a preliminary report. Clin Orthop 174:127-132, 1983

6. Kim NH, Lee HM, Chung IH, Kim HJ, Kim SJ: Morphometric study of the pedicles of thoracic and lumbar vertebrae in Koreans. Spine (Phila Pa 1976) 19:1390-1394, 1994

7. Ruetten S, Komp M, Merk H, Godolias G: Surgical treatment for lumbar lateral recess stenosis with the full-endoscopic interlaminar approach versus conventional microsurgical technique: a prospective, randomized, controlled study. J Neurosurg Spine 10:476-485, 2009

8. Tenenbaum S, Arzi H, Herman A, Friedlander A, Levinkopf M, Arnold PM, et al: Percutaneous posterolateral transforaminal endoscopic discectomy: clinical outcome, complications, and learning curve evaluation. Surg Technol Int 21:278-283, 2011

9. Thomé C, Barth M, Scharf J, Schmiedek P: Outcome after lumbar sequestrectomy compared with microdiscectomy: a prospective randomized study. J Neurosurg Spine 2:271278, 2005

10. Tokuhashi Y, Matsuzaki H, Uematsu Y, Oda H: Symptoms of thoracolumbar junction disc herniation. Spine (Phila Pa 1976) 26:E512-E518, 2001

11. Urrutia VE, Eliozondo ORE, De la Garza CO, Guzman LS: Morphometry of pedicle and vertebral body in a Mexican population by CT and fluoroscopy. Int J Morphol 27:12991303,2009

12. Wang J, Zhou Y, Zhang ZF, Li CQ, Zheng WJ, Huang B: Disc herniation in the thoracolumbar junction treated by minimally invasive transforaminal interbody fusion surgery. J Clin Neurosci 21:431-435, 2014

13. Yeom KS, Choi YS: Full endoscopic contralateral transforaminal discectomy for distally migrated lumbar disc herniation. J Orthop Sci 16:263-269, 2011

\section{Disclosures}

The authors report no conflict of interest concerning the materials or methods used in this study or the findings specified in this paper.

\section{Author Contributions}

Conception and design: Telfeian, Jasper. Acquisition of data: Telfeian, Jasper. Analysis and interpretation of data: Telfeian, Jasper, Gokaslan. Drafting the article: Telfeian, Jasper. Critically revising the article: all authors. Reviewed submitted version of manuscript: all authors. Approved the final version of the manuscript on behalf of all authors: Telfeian. Statistical analysis: Jasper. Administrative/technical/material support: Telfeian, Jasper, Gokaslan. Study supervision: Telfeian.

\section{Supplemental Information \\ Videos \\ Video 1. https://vimeo.com/148102334.}

\section{Correspondence}

Albert Telfeian, Department of Neurosurgery, Rhode Island Hospital, 593 Eddy St., Providence, RI 02903.email: atelfeian@ lifespan.org. 